\title{
Eficiência da Armadilha "R. Bianco" para Captura do Percevejo Leptoglossus zonatus Dallas (Hemiptera: Coreidae), na Cultura do Milho
}

\author{
Marliton Rocha Barreto ${ }^{\bowtie}$ \& Ledonir Geovani da Silva
}

Universidade Federal de Mato Grosso, e-mail: mrb.ufmt@gmail.com (Autor para correspondência ${ }^{\bowtie}$ ), geovani.gil@hotmail.com.

\section{EntomoBrasilis 9 (2): 84-88 (2016)}

Resumo. O milho é o cereal com maior índice de consumo, tanto industrializado como in natura, atualmente. É o grão com maior volume de produção, sendo o Brasil o terceiro maior produtor, dessa maneira, faz-se necessário uma atenção especial voltada ao monitoramento e controle de certos invasores que comprometem sua produtividade. Dentre as pragas que podem afetar essa produtividade destacamos os percevejos (Hemiptera: Heteroptera), mais especificamente o percevejo-do-milho (Leptoglossus zonatus Dallas), que tem se mostrado uma importante praga para a cultura do milho. O presente trabalho teve por objetivo avaliar a eficiência de armadilhas caseiras do tipo "R. Bianco" na captura desse percevejo. Os experimentos foram conduzidos no Sitio Nossa Senhora Aparecida, localizado no município de Sinop, MT. As armadilhas foram divididas em 10 abertas e 10 fechadas e avaliadas com e sem isca por 20 dias. O delineamento utilizado foi o inteiramente casualizado (DIC) em esquema fatorial $2 \times 2 \times 2$ com oito tratamentos e cinco repetições. As armadilhas sem iscas demonstraram maior eficiência, apresentando média superior na captura do percevejo em relação às armadilhas com isca. As armadilhas fechadas, em comparação com as abertas, demonstraram maior eficácia na retenção do inseto. $\mathrm{O}$ efeito da borda foi pouco representativo nesse experimento. Portanto, a presença da isca não influenciou na atração do inseto e os tratamentos submetidos ao teste sem isca apresentaram maiores quantidades e maior eficiência na captura de insetos. Levando a crer o efeito de atração do sal pelo percevejo e a localização de cada armadilha pouco influenciou na captura dos insetos.

Palavras-chave: Armadilha caseira; milho; monitoramento; percevejo-do-milho; pragas.

\section{Trap Efficiency "R. Bianco" for Capture the insect Leptoglossus zonatus Dallas (Hemiptera: Coreidae) in Maize Crop}

Abstract. Maize is the cereal with the highest consumption in both industrialized as fresh nowadays. It is the grain with higher volume production, Brazil is the third largest producer, need special attention focused on the monitoring and control of invaders who risk their productivity. Among the pests that can affect the productivity highlight the insects (Hemiptera: Heteroptera), more specifically the bedbugs corn (Leptoglossus zonatus Dallas), which has been an important pest for corn crops. Thus, this study aimed to evaluate the effectiveness of homemade traps R. Bianco in control of this insect. The experiments were conducted in Sitio Nossa Senhora Aparecida, in the municipality of Sinop, MT. Traps were divided into 10 open and 10 closed which were evaluated with and without baits by 20 days. The design was completely randomized (DIC) in a 2x2x2 factorial with eight treatments and five repetitions. Traps without baits showed greater efficiency, with an average higher in catching the bugs regarding the traps with bait. The trap closed as compared with the open, demonstrated greater efficacy in retaining the insect. The effect of edge was little representative in this experiment. Therefore, the presence of bait did not influence the insect attraction and treatments submitted to the bait without test showed higher quantities and more efficient insect capture. Leading us to believe the attraction effect of salt by bedbug and the location of each trap had little influence in the capture of insects.

Keywords: Bedbugs corn; corn; homemade traps; monitoring; pests.

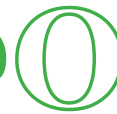
milho (Zea mays L.) é um dos alimentos mais consumidos no mundo (CRUZ et al. 2008). Em 2014/15 a área de milho plantada no Brasil foi de 15.743 .700 ha, destes 6.156.100 ha são de milho primeira safra e 9.587.60o ha são de segunda safra (milho safrinha). A produção brasileira de milho segunda safra, foi de 54.485.100 ton., com Mato Grosso representando 37,26\% desse montante (ConAB 2015).

Uma forma de cultivo que vem ganhando espaço é a do milho verde, que quando comparado com o milho grão, por ser colhido mais cedo, possui maiores teores de açúcar e menor teor de amido (Matos 2007). Embora a produção de milho verde seja menor que a de grãos secos, no Brasil seu cultivo cresce a cada ano devido ao valor agregado ao produto e seus derivados (VIEIRA 2007). O milho verde é produzido em pequenas propriedades sob sistemas de irrigação ainda limitados, possuem grande valor nutritivo podendo ser utilizado no consumo humano bem como na indústria de conserva ou enlatados (NASCIMENTO 2012).
Vários insetos atacam as sementes, raízes e plântulas (Plantas jovens) do milho após a semeadura. $\mathrm{O}$ tipo de ataque reduz o número de plantas na área cultivada e o potencial produtivo da lavoura (Lima et al. 2016). Dentre eles, o ataque de percevejos durante o período vegetativo tem ganhado importância nos últimos anos no Brasil, principalmente nos cultivos de milho e sorgo. No entanto, as maiores populações são encontradas nos meses de outono e inverno, logo após a colheita da soja, mais comumente em sistemas de plantio direto (RodRIGUES 2011). RozAGomes et al. (2011), caracterizou as injúrias causadas por quatro espécies de Hemiptera: Pentatomidae: Dichelops furcatus Fabr., Nezara veridula Linnaeus, Euschistos heros Fabr. e Dichelops melacanthus Dallas, em plântulas de milho. Entretanto, outra praga que ocorre na cultura do milho e até pouco tempo era considerado como praga secundária é o Leptoglossus zanatus Dallas, Coreidae (MoreIRA \& ARAGÃo 2009).

A ocupação de novas áreas geográficas para o plantio do cereal resultou em alterações nas condições ambientais favorecendo a 
T incidência de pragas antes consideradas de pouca importância (SouzA \& BALDin 2009). O percevejo L. zonatus é um inseto polífago, encontrado em quase toda a América, atacando plantas frutíferas, gramíneas e leguminosas acarretando prejuízos principalmente ao milho, sorgo e laranja (BRAGA 2013), assim como em romã, maracujá, citros, acerola, amora, manga e goiabeira (Matrangolo \& WAQUIL 1993; RAGa et al. 1995; RodrigUeS Netto \& Guilhem 1996; Henne et al. 2003; Pires et al. 2012; Souza Filho \& Costa 2014). Sua ocorrência pode ser observada na cultura do milho do meio para o final do ciclo da planta, onde tanto os adultos como as ninfas preferem se alimentar das espigas, principalmente dos grãos leitosos em formação (BRAGA 2013), causando murchamento e apodrecimento, favorecendo a possível contaminação por fungos como Fusarium, Penicillium e Cephalosporium (MonsANTO 2014).

Segundo Pires et al. (2011), devido ao período entressafra, quando as áreas cultivadas não estão ocupadas com soja e milho, $L$. zonatus tem sido relatado em carambola, inserindo o estilete para alimentação causando danos a superfície do fruto, permitindo que outros insetos ataquem o fruto através do orifício provocado pelo percevejo, tornando o fruto inviável ao consumo. Há relatos ainda, de que, L. zonatus tem se expandido cada vez mais para espécies frutíferas nas entre safras, dando uma ideia de que tais alternativas de alimentos superem a escassez de milho safrinha, mantendo a população de insetos em níveis significativos (PIRES et al. 2012).

O presente trabalho objetivou avaliar a eficiência das armadilhas do tipo R. Bianco, na captura do percevejo L. zonatus na cultura do milho.

\section{MATERIAL E MÉTODOS}

O presente trabalho foi conduzido no Sítio Nossa Senhora Aparecida ( $11^{\circ} 50^{\prime} 32.2$ "S, $55^{\circ} 21^{\prime} 02.6$ ” W), localizado na estrada Elizabeth, comunidade Branca de Neve, a cerca de $30 \mathrm{~km}$ de Sinop, MT no período de 02/02/2013 à 12/05/2013. Foram utilizados cinco talhões de um hectare cada, onde o milho verde (variedade AG1051) juntamente com cana de açúcar foram plantados no período chuvoso, de novembro a abril. A temperatura média variou entre $25^{\circ} \mathrm{C}$ e $40^{\circ} \mathrm{C}$.

Foram confeccionadas 20 armadilhas do tipo "R. Bianco" (adaptadas) com garrafas pet, divididas em 10 abertas (Figura 1A) e 10 fechadas (Figura 1B). Em paralelo, pesou-se $600 \mathrm{~g}$ de grãos de soja seca que foram colocadas em um recipiente com água limpa por 10-15 min.. Em seguida foi escoada a água, adicionada uma colher (chá) de sal de cozinha, homogeneizado, e o volume de grãos total foi dividido por 20 partes (iscas) iguais e adicionados às garrafas. Posteriormente, em 10 armadilhas (cinco abertas $\mathrm{e}$ cinco fechadas) foi adicionado o inseticida Connect (Bayer), do grupo químico dos Neonicotinóides (Imidacloprido) e Piretróides (Beta-ciflutrina). As iscas foram colocadas na área experimental após preparadas e a avaliação foi realizada $24 \mathrm{~h}$ após, conforme preconizado por BIANCO (1991).

A avaliação foi realizada por talhão e ocorreu na fase de pendoamento (estádio VT) até a colheita (estádio R3). Em cada área foram instaladas 20 armadilhas, numeradas e dispostas em duas linhas distintas nas laterais do talhão e uma linha no centro. Cada talhão foi avaliado por aproximadamente 20 dias e, após a colheita, as armadilhas eram transferidas para a próxima área em que se encontrava na fase de pendoando.

Depois de cada avaliação ( 24 h após), as iscas foram retiradas e as armadilhas foram mantidas no campo por mais $24 \mathrm{~h}$. Nesta etapa, o objetivo era avaliar se apenas com os restos de sal e agroquímico elas ainda continuavam atrativas e a avaliação se deu pelo número de insetos (vivos e mortos) presentes no interior de cada armadilha e pela eficiência na captura conforme o tipo da armadilha (aberta ou fechada).

O delineamento utilizado foi o inteiramente casualizado (DIC), com oito tratamentos e cinco repetições em esquema fatorial $2 \mathrm{x}$ $2 \times 2$, sendo utilizado, diferentes modelos de armadilhas (aberta e fechada); presença ou ausência de agroquímico e presença ou ausência de iscas. Os dados obtidos foram submetidos à análise de variância e as médias, quando significativas, submetidas ao teste de Scott-Knott pelo programa de análises estatísticas ASSISTAT, versão 7.7 beta (SiLVA \& AZEVEDo 2009).

\section{RESULTADOS E DISCUSSÃO}

Os dados obtidos pela quantidade de armadilhas que atraíram L. zonatus demonstram que, na interação entre iscas $\mathrm{x}$ modelos de armadilha houve diferença significativa, onde as modelos abertas e fechadas sem isca foram superiores em relação aos modelos abertas e fechadas com isca, dando uma ideia de que a soja empregada dentro de cada armadilha não seja atrativa para a captura do inseto. Entretanto, a armadilha fechada sem isca demonstrou maior atratividade do percevejo em relação ao outro modelo e isca testada, pois ao adentrar na armadilha o inseto não consegue sair, sendo de fácil escapatória para o inseto, constatando efeito atrativo do sal (Tabela 1).

As interações em função de iscas versus agroquímico e modelo versus agroquímico não demonstraram diferenças significativas entre si, não sendo possível uma analise satisfatória de seu efeito atrativo pelo inseto, o mesmo ocorreu na análise da interação

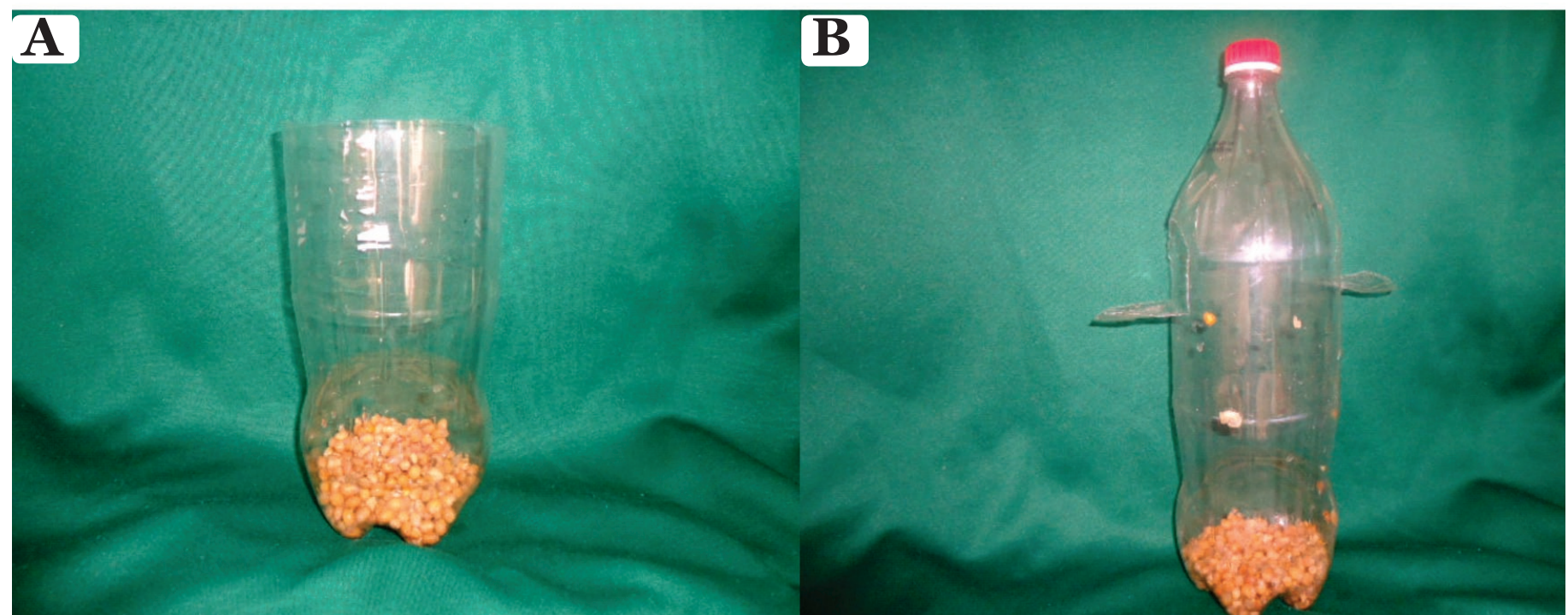

Figura 1. Armadilhas tipo R. Bianco aberta (A) e fechada (B) com isca. Foto: Ledonir Geovani da Silva 
iscas versus modelo versus agroquímico onde não houve diferença entre os fatores.

Tabela 1. Número de armadilhas que capturaram L. zonatus em função das interações entre iscas x modelos; iscas $\mathrm{x}$ agroquímico; modelos $\mathrm{x}$ agroquímico; iscas x modelos $\mathrm{x}$ agroquímico.

\begin{tabular}{|c|c|c|}
\hline \multirow{2}{*}{ Iscas } & \multicolumn{2}{|c|}{ Modelos } \\
\hline & Aberta & Fechada \\
\hline Com Isca & $8,30 \mathrm{~b} \mathrm{~A}$ & $8,40 \mathrm{~b} \mathrm{~A}$ \\
\hline Sem Isca & 11,20 a $B$ & 15,10 a $\mathrm{A}$ \\
\hline \multirow{2}{*}{ Iscas } & \multicolumn{2}{|c|}{ Agroquímico } \\
\hline & Com Agroquímico & Sem Agroquímico \\
\hline Com Isca & 8,70 a A & 8,00 a A \\
\hline Sem Isca & 14,00 a A & 12,30 a A \\
\hline \multirow{2}{*}{ Modelos } & \multicolumn{2}{|c|}{ Agroquímico } \\
\hline & Com Agroquímico & Sem Agroquímico \\
\hline Aberta & 10,80 a A & 8,70 a A \\
\hline Fechada & 11,90 a A & 11,60 a $\mathrm{A}$ \\
\hline \multirow{2}{*}{ Iscas x Modelos } & \multicolumn{2}{|c|}{ Agroquímico } \\
\hline & Com Agroquímico & Sem Agroquímico \\
\hline Com Isca $x$ Aberta & 9,20 a A & 7,40 a A \\
\hline Com Isca x Fechada & 8,20 a A & 8,60 a $\mathrm{A}$ \\
\hline Sem Isca x Aberta & 12,40 a A & 10,00 a A \\
\hline Sem Isca $x$ Fechada & 15,60 a $A$ & 14,60 a $A$ \\
\hline CV (\%) & & 18,72 \\
\hline
\end{tabular}

Médias seguidas de letras minúsculas iguais na coluna e maiúsculas na linha não diferem significativamente pelo teste de Scott-Knott ao nível de $5 \%$ de probabilidade.

Dados semelhantes obtiveram CorrêA-Ferreira \& Peres (2006) com armadilhas contendo urina bovina + sal, observando quantidades superiores de fêmeas em relação aos machos de várias espécies de percevejos. A utilização de sal de cozinha $(\mathrm{NaCl})$ ou Ureia, auxilia na redução de metade das doses dos inseticidas empregados no controle do percevejo-do-milho. A ação do cloreto de sódio não é de um atraente, mais sim de um estimulante alimentar, ocorrendo assim um maior contato entre o inseto e o inseticida. Sendo assim, quando aplicado na mistura do inseticida afeta o comportamento dos percevejos, aumentando o tempo de permanência do inseto sobre o alimento (PAnizzi \& Oliveira 1993; CorrêA-Ferreira \& PanizZi 1999).

BIANCO (2005) recomenda que até duas armadilhas com insetos possa ser considerado um nível de risco baixo, não justificando medidas de controle agressivas; de três a cinco armadilhas com inseto apresenta nível de risco moderado, onde o uso de inseticidas podem ser empregados, porém acompanhado de um monitoramento adequado; acima de cinco armadilhas com inseto há um nível de risco alto para a cultura, onde medidas de controle devem ser tomadas atentando para o uso mínimo possível de agrotóxicos, dando preferência a modelos de controle biológico.

A eficiência das armadilhas na captura do percevejo-do-milho, quando submetidas aos experimentos com isca e sem isca, observou-se que não houve diferença entre os tratamentos com iscas, não diferindo estatisticamente umas das outras. Conforme observado na Tabela 2, houve diferença significativa no fator iscas versus modelos de armadilhas, onde a armadilha fechada sem isca mostrou-se mais eficiente na captura do inseto, constatando então, sua eficiência como armadilha atrativa para o percevejo L. zonatus.

HoFFMANN-CAMPO et al. (2003), testou vários tipos de armadilhas diferentes para captura de percevejo, entre elas cita-se a armadilha fechada simples de garrafa pet com urina bovina mais sal de cozinha, deixadas por sete dias no campo, coletando 85 percevejos por armadilha. Observou-se que a maioria dos percevejos coletados foi de adultos, o qual também foi constatado visualmente neste trabalho. Fato esse atribuído ao fato de serem alados e se dispersarem mais facilmente na área.

PANizzi (2004) relatou o comportamento de L. zonatus em armadilhas com garrafas pet deixadas a campo, separados a cada $10 \mathrm{~m}$. Com o passar do tempo observou um aumento na população de insetos no primeiro dia $(\mathrm{N}=90)$, decrescendo essa população com o passar dos dias em, aproximadamente 60\% a partir do quarto dia, chegando a $10 \%$ no oitavo dia $(\mathrm{N}=9)$, sugerindo uma defesa territorial ou reconhecimento e uma possível aceitação do objeto como parte de seu habitat.

Tabela 2. Número de L. zonatus capturados em função das interações entre iscas x modelos; iscas $\mathrm{x}$ agroquímico; modelos $\mathrm{x}$ agroquímico; iscas $\mathrm{x}$ modelos $\mathrm{x}$ agroquímico.

\begin{tabular}{|c|c|c|}
\hline \multirow{2}{*}{ Iscas } & \multicolumn{2}{|c|}{ Modelos } \\
\hline & Aberta & Fechada \\
\hline Com Isca & 4,0486 b A & 4,1973 b A \\
\hline Sem Isca & 5,2504 a B & 7,1230 a $\mathrm{A}$ \\
\hline \multirow{2}{*}{ Iscas } & \multicolumn{2}{|c|}{ Agroquímico } \\
\hline & Com Agroquímico & Sem Agroquímico \\
\hline Com Isca & $4,1082 \mathrm{a} \mathrm{A}$ & 4,1377 a A \\
\hline Sem Isca & 6,8531 a A & 5,5203 a A \\
\hline \multirow{2}{*}{ Modelos } & \multicolumn{2}{|c|}{ Agroquímico } \\
\hline & Com Agroquímico & Sem Agroquímico \\
\hline Aberta & 4,7979 a A & 4,5010 a $\mathrm{A}$ \\
\hline Fechada & 6,1633 a A & 5,1569 a A \\
\hline \multirow{2}{*}{ Iscas $\mathrm{x}$ Modelos } & \multicolumn{2}{|c|}{ Agroquímico } \\
\hline & Com Agroquímico & Sem Agroquímico \\
\hline Com Isca x Aberta & 3,7927 a A & 4,3044 a A \\
\hline Com Isca $x$ Fechada & 4,4237 a A & 3,9709 a A \\
\hline Sem Isca $x$ Aberta & 5,8032 a A & 4,6976 a A \\
\hline Sem Isca $x$ Fechada & 7,9030 a A & 6,3429 a A \\
\hline CV (\%) & & 23,90 \\
\hline
\end{tabular}

Médias seguidas de letras minúsculas iguais na coluna e maiúsculas na linha não diferem significativamente pelo teste de Scott-Knott ao nível de $5 \%$ de probabilidade. Médias transformadas em $\mathrm{X}=(\mathrm{X})^{1 / 2}$.

As demais interações, iscas versus agroquímico e modelo versus agroquímico e a interação entre iscas versus modelo pelo agroquímico, não diferiram significativamente pelo método estatístico adotado. Entretanto, a presença do agroquímico dentro de cada armadilha pode auxiliar na morte do inseto no momento de sua captura sem afetar a atração do percevejo pela armadilha. Ainda conforme verificado na Tabela 2, a ocorrência de percevejos nas armadilhas sem iscas fechadas foi superior às armadilhas abertas, em todos os fatores onde os modelos foram testados. Assim, podemos concluir que as armadilhas fechadas dificultaram a saída do inseto do interior, consequentemente a maioria abatida pelos vestígios do agroquímico aplicado anteriormente com a soja.

Em trabalho semelhante a partir de garrafas pet, Lima Filho et al. (2014) testou um novo modelo eficiente para captura de insetos em uma área de arbustos. Como isca utilizou-se de cerveja sem álcool, sucos de abacaxi, caju e maracujá e gotas de detergente neutro. O maior número de insetos ocorreu na armadilha contendo cerveja sem álcool (122 insetos) e menor número no suco de maracujá (56 indivíduos). 
Em seus estudos Pires et al. (2006) utilizou 4 modelos de armadilhas diferentes iscadas com feromônio para captura do percevejo marrom. A armadilha modelo 4, composta por garrafa pet com 4 perfurações ao redor, foi que apresentou maior eficiência, cerca de $98 \%$ na retenção do percevejo. Silva et al. (2014), relataram que armadilhas com feromônio sexual capturaram preferencialmente fêmeas de $E$. heros, o que representa 77 e $82 \%$ dos insetos retidos nas armadilhas com a formulação em septos de borracha e lure, respectivamente.

Nesse experimento, ainda pode-se avaliar a ocorrência de percevejos capturados nas bordas e no meio dos talhões. Entretanto, houve abundância do inseto capturado nas duas localizações, tal fato pode ter ocorrido pela presença de plantas daninhas que se instalaram até o final do desenvolvimento do milho. Portanto, a localização das armadilhas dentro de cada talhão não influenciou na captura do percevejo. HoFFMANN-CAMPO et al. (2003) afirma que as armadilhas devem ser normalmente instaladas na bordadura das lavouras, desde o período inicial da cultura, com a finalidade de capturar a população colonizante. Entretanto, a instalação das armadilhas neste experimento, ocorreu durante o pendoamento do milho, portanto a cultura já havia sido colonizada pelo inseto, resultando também na pouca diferença entre coletados no meio e nas bordas da área.

Portanto, a presença da isca não influenciou na atração do inseto, ou seja, a soja empregada dentro de cada armadilha não atuou como atrativo para o percevejo, podendo ainda ter mascarado o efeito atrativo do sal. Os tratamentos submetidos ao teste sem isca apresentaram maiores quantidades e maior eficiência na captura de insetos, levando a crer o efeito de atração do sal pelo percevejo e a localização de cada armadilha pouco influenciou na captura dos insetos, sendo então, eficiente tanto na borda como no meio da área experimental, portanto a disposição das armadilhas pode ser feita de forma aleatória.

\section{AGRADECIMENTOS}

Ao Sr. Edilson Smiderle por ceder a propriedade para realização dos experimentos.

\section{REFERÊNCIAS}

Bianco, R., 1991. Pragas do milho e seu controle, p. 187-221. In: A cultura do milho no Paraná. Londrina, IAPAR, Circular 68

Bianco, R., 2005. Manejo de Pragas do Milho em Plantio Direto, p. 8-17. In: Instituto Biológico de São Paulo. (Org.). Encontro de Fitossanidade de Grãos. Campinas, Emopi editora e gráfica. Disponível em: <http://www.biologico.sp.gov.br/ rifib/XI RIFIB/bianco.PDF>. [Acesso em: 17.vi.2015.

Braga,I.M.S., 2013 Ocorrência edinâmica populacional deinsetospragas e predadores associados às culturas de nabo forrageiro e milho em sistemas de plantio direto, em Chapadão do Sul, MS. Dissertação (Mestrado em Agronomia - Entomologia Agrícola) - Programa de Mestrado em Entomologia Agrícola da Faculdade de Ciências Agrárias e Veterinárias, Jaboticabal, 40 f. Disponível em: < http://acervodigital.unesp.br/handle/ unesp $/ 172459$ ? locale $=$ pt BR $>$. [Acesso em: 17.vi.2015.

CONAB - Companhia Nacional de Abastecimento. 2015. Acompanhamento da safra brasileira: grãos. Décimo segundo levantamento. Brasília: Conab, 2015. Disponível em: <http://www.conab.gov.br/OlalaCMS/uploads/ arquivos/15 $09 \quad 11 \quad 10 \quad 42 \quad 03$ boletim graos setembro 2015.pdf >. [Acesso em: 17.vi.2015].

Corrêa-Ferreira, B.S. \& A.R. Panizzi, 1999. Percevejos da soja e seu manejo. Londrina: Embrapa-CNPSo (Circular técnica, 24), 45 p. Disponível em: <https://www.agencia.cnptia. embrapa.br/Repositorio/circTec24 00og4vbbaaq02wx5ok odklaos1mgl51b.pdf>. [Acesso em: 15.vi.2015].

Corrêa-Ferreira, B.S. \& W.A.A. Peres, 2006. Alternativas para o manejo de percevejos-pragas (Hemiptera: Pentatomidae) em sistema de soja orgânica. Revista Brasileira de Agroecologia. 1:
649-652. Disponível em: <http://www.agroecologiaemrede. org.br/upload/arquivos/P202 2005-07-25 165946160. pdf $>$. [Acesso em: 15.vi.2015].

Cruz, J.C., D. Karam, M.A.R. Monteiro \& P.C. Magalhães, 2008. A cultura do milho. Sete Lagoas, Embrapa milho e sorgo, 517 p. Disponível em: <http://livraria.sct.embrapa.br/liv resumos/pdf/00062780.pdf $>$. [Acesso em: 10.vi 2015].

Henne D.C., S.J. Johnson \& W.J. Bourgeois, 2003. Pest status of leaf-footed bugs (Heteroptera: Coreidae) on citrus in Louisiana. Proceedings of the Annual Meeting of the Florida State Horticultural Society, 116: 240-241. Disponível em: <http://fshs.org/proceedings-o/2003-vol-116/240-241. pdf $>$. [Acesso em: 12.vi.2015].

Hoffmann-Campo, C.B., D.R. Sosa-Gomez, C.S. Corrêa-Ferreira \& D.L. Gazzoni, 2003. Alternativas potenciais para uso no manejo de pragas da soja. In: Corrêa-Ferreira, B.S. Soja orgânica: alternativas para o manejo dos insetos-pragas. Londrina: Embrapa CNPSo, p. 65-83. Disponível em: <https://www.embrapa.br/soja/busca-de-publicacoes/-/ publicacao/463696/soja-organica-alternativas-para-omanejo-dos-insetos-pragas >. [Acesso em: 15.vi.2015].

Lima Filho, J.A.; A.G.C. Oliveira, O.O. Freire, B.M.G. Bezerra \& V. M. Santos, 2014. Captura de insetos utilizando um novo modelo de armadilha com área do IFPB campus Campina Grande, PB. Revista Gaia Scientia, 8: 74-79. Disponível em: <http://oaji.net/articles/2014/1214-1409593124.pdf >. [Acesso em: 10.vi.2015].

Lima, B.V., B.S. Caetano, G.G. de Souza, M.T. Spontoni \& L.C.D. de Souza, 2016. Pragas da cultura do Milho. Revista Conexão Eletrônica, 13: 1-15.

Matos, E.H.S.F., 2007. Cultivo do milho verde. Dossiê técnico. Disponível em: <http://www.agrolink.com.br/downloads/ Cultivo\%20do\%20Milho\%2oVerde.pdf $>$. [Acesso em: 05.X.2014].

Matrangolo, W.J.R. \& J.M. Waquil, 1993. Biology of Leptoglossus zonatus (Dallas) (Hemiptera: Coreidae) fed on maize and sorghum. Anais da Sociedade Entomológica do Brasil, 23: 419-423.

Monsanto, 2014. Manual de pragas do milho, da soja e do algodão. Disponível em: <http://www.monsanto.com/global/br/ noticias/pages/monsanto-lanca-manual-de-pragas-onlinegratuito.aspx $>$. [Acesso em: 05.x.2014].

Moreira, H.J.C. \& F.D. Aragão, 2009. Manual de pragas do milho. Campinas, FMC, 144 p. Disponível em: <http://www. agrolink.com.br/downloads/manual\%20de\%2opragas\%20 do\%20milho.pdf>. [Acesso em: 06.x.2014].

Nascimento, F.N., 2012. Características agronômicas do milho verde sob diferentes regimes hídricos. Dissertação (Mestrado em agronomia - área de concentração em produção vegetal) - Programa de Pós-graduação em Agronomia do Centro de Ciências Agrárias da Universidade Federal do Piauí, Teresina, 81 p. Disponível em: <http://ainfo.cnptia.embrapa.br/ digital/bitstream/item/70578/1/Dissertacao-Fabio.pdf $>$. [Acesso em: 01.xi.2014].

Panizzi, A.R. \& N. Oliveira, 1993. Atração de cloreto de sódio (sal de cozinha) aos percevejos-pragas da soja. In: EMBRAPA. Centro Nacional de Pesquisa de Soja (Londrina, PR). Resultados de pesquisa de soja 1989/90, EMBRAPA-CNPSo. Documentos, 58. Londrina, p. 71-76.

Panizzi, A.R., 2004. A possible territorial or recognition behavior of Leptoglossus zonatus (Dallas) (Heteroptera, Coreidae). Revista Brasileira de Entomologia, 48: 577-579. DOI: http:// dx.doi.org/10.1590/S0085-56262004000400022.

Pires, C.S.S., E.R. Sujii, F.G.V. Schmidt, P.H.G. Zarbin, J.R.M. Almeida \& M. Borges, 2006. Potencial de uso de armadilhas iscadas com o feromônio sexual do percevejo marrom, Euschistus heros (Heteroptera: Pentatomidae), para o monitoramento populacional de percevejos praga da soja. Manejo Integrado de Plagas y Agroecologia (Costa Rica), 77: 70-77. 
Pires, E.M., S. Ruffato, C.L.M. Manica, M.A. Soares \& M.C. Lacerda, 2012. Novas Plantas Hospedeiras para o Percevejo Fitófago Leptoglossus zonatus (Dallas) (Hemiptera: Coreidae). EntomoBrasilis, 5: 249-252. DOI: http://dx.doi.org/10.12741/ebrasilis.v5i3.236.

Pires, E.M., S.M. Bonaldo, J.A.M. Ferreira, M.A. Soares \& S. Candan, 2011. New record of Leptoglossus zonatus (Heteroptera: Coreidae) attacking starfruit (Averrhoa carambola) in Sinop, Mato Grosso, Brazil. EntomoBrasilis, 4: 33-35. DOI: http://dx.doi.org/10.12741/ebrasilis.v4i1.114.

Raga, A.C., J. Piza \& M.F. Souza Filho, 1995. Ocorrência e danos de Leptoglossus zonatus (Dallas) (Heteroptera: Coreidae) em Romã, Punica granatum L., em Campinas, São Paulo. Anais da Sociedade Entomológica do Brasil, 24: 183-185.

Rodrigues Netto, S.M. \& D.J. Guilhem, 1996. Ocorrência de Leptoglossus zonatus (Dallas, 1852) (Hemiptera: Coreidae) em maracujazeiro (Passiflora edulis f. flavicarpa). Arquivos do Instituto Biológico, 63: 85-86.

Rodrigues, R.B., 2011. Danos percevejo barriga-verde Dichelops melacanthus (Dallas, 1851) (Hemiptera: Pentatomidae) na cultura do milho. Dissertação (Mestrado em agronomia área de concentração em produção vegetal) - Programa de Pós-graduação em Agronomia, Universidade Federal de Santa Maria, Santa Maria, 105 p. Disponível em: <http://livroso1. livrosgratis.com.br/cp092015.pdf $>$. [Acesso em: 17.vi.2015].

Roza-Gomes, M.F., J.R. Salvadori, P.R.V.S. Pereira \&A.R. Panizzi, 2011. Injúrias de quatro espécies de percevejos pentatomídeos em plântulas de milho. Ciência Rural. 41: 1115-1119. DOI: http://dx.doi.org/10.1590/s0103-84782011005000081.

Silva, F.A.S. \& C.A.V. Azevedo, 2009. Principal Components Analysis in the Software Assistat-Statistical Attendance. In: World Congress on Computers in Agriculture, 7, RenoNV-USA: American Society of Agricultural and Biological Engineers. DOI: http://dx.doi.org/10.13031/2013.29066.
Silva, V.P. da, M.J.B. Pereira, L.M. Vivan, M.C. Blassioli-Moraes, R.A. Laumann \&M. Borges, 2014. Monitoramento do percevejo marrom Euschistus heros (Hemiptera: Pentatomidae) por feromônio sexual em lavoura de soja. Pesquisa Agropecuária Brasileira, 49: 844-852. DOI: http://dx.doi.org/10.1590/ s0100-204X2014001100003.

Souza Filho, M.F. \& V.A. Costa, 2014. Manejo integrado de pragas da goiabeira, 33 p. Disponível em: <http://www. nutricaodeplantas.agr.br/site/ensino/pos/Palestras William/Livrogoiaba pdf/g MIPpragas.pdf $>$. [Acesso em 12.X.2014].

Souza, E.S. \& E.L.L. Baldin, 2009. Preferência alimentar easpectos biológicos de Leptoglossus zonatus Dallas, 1852 (Hemiptera: Coreidae) em diferentes genótipos de milho. Boletín de Sanidad Vegetal Plagas, 35: 175-185. Disponível em: <https:// dialnet.unirioja.es/servlet/articulo? codigo $=3105707>$. [Acesso em: 17.vi.2015.

Vieira, M.A., 2007. Cultivares e população de plantas na produção de milho verde. Dissertação (Mestrado em Agronomia - área de concentração em produção vegetal) - Departamento de Fitotecnia e Fitossanitarismo da Universidade Federal do Paraná, Curitiba, 95 p. Disponível em: <http://acervodigital. ufpr.br/bitstream/handle/1884/14826/marcelovieirafinal. pdf? sequence $=1>$. [Acesso em: 17.vi.2015].

\section{Recebido em: 15.v.2015}

Aceito em: 20.vi.2016

$* * * * * * * * *$

\section{Como citar este artigo:}

Barreto, M.R. \& L.G. Silva, 2016. Eficiência da Armadilha "R. Bianco" para Captura do Percevejo Leptoglossus zonatus Dallas (Hemiptera: Coreidae), na Cultura do Milho. EntomoBrasilis, 9 (2): 84-88.

Acessível em: doi:10.12741/ebrasilis.vgi2.531
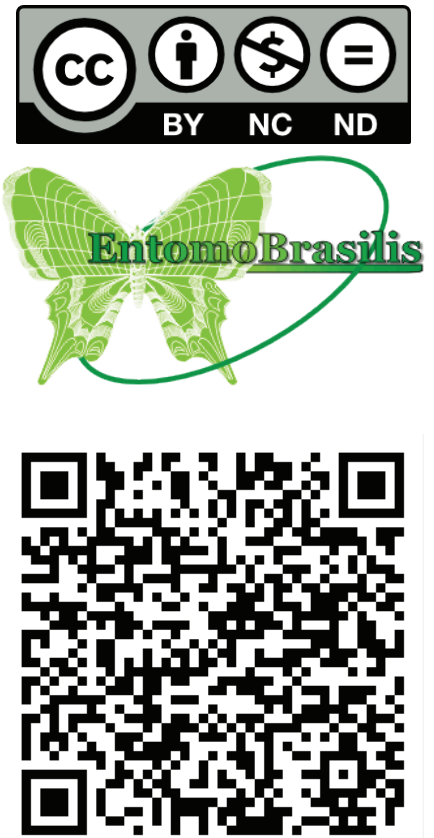